\title{
Concepciones sobre la reproducción sexual en Filón de Alejandría y sus antecedentes científicos griegos
}

\author{
Laura PÉREZ \\ Universidad Nacional de La Pampa. Argentina / \\ Consejo Nacional de Investigaciones Científicas y Técnicas \\ lau_perez75@hotmail.com
}

Recibido: 25-11-2014

Aceptado: 15-12-2014

\section{RESUMEN}

Este trabajo se propone analizar la influencia de los conocimientos científicos griegos producidos en el campo de los estudios biológicos, fisiológicos y médicos sobre el filósofo judeohelenista Filón de Alejandría, a través del examen de sus concepciones sobre el funcionamiento de la reproducción sexual humana y, en particular, sobre la cuestión de la participación y la función de hombres y mujeres en el proceso de gestación. En primer lugar, estudiamos las diversas teorías acerca de esta temática que circulaban en el mundo griego: las ideas tradicionales expresadas en la tragedia, las teorías de los filósofos presocráticos, de la medicina hipocrática y de Aristóteles. Luego, analizamos las ideas que Filón expresa sobre este asunto, indagamos cuál o cuáles de las teorías griegas ejerció mayor grado de influencia en su pensamiento e intentamos explicar los motivos por los que Filón se apropia de algunas de estas teorías mientras descarta otras.

Palabras clave: Reproducción sexual, presocráticos, medicina hipocrática, Aristóteles, Filón de Alejandría.

\begin{abstract}
This work intends to analyze the influence of the Greek scientific knowledge produced in the fields of biological, physiological and medical studies over the Hellenistic Jewish philosopher Philo of Alexandria, through an examination of his conceptions about the process of human sexual reproduction and, in particular, about the issue of the participation and role of men and women in gestation. In the first place, we study the different theories about this subject which circulated in the Greek world: the traditional views expressed in tragedy, the theories of the pre-Socratic philosophers, of Hippocratic medicine and of Aristotle. Then, we analyze the ideas expressed by Philo about this matter, we investigate which of the Greek theories exerted greater influence over his thought and we try to explain why Philo appropriates some of these theories while discards others.
\end{abstract}

Keywords: sexual reproduction, pre-Socratics, Hippocratic medicine, Aristotle, Philo of Alexandria.

\section{SUMARIO}

1. De la tragedia a los primeros filósofos, 2. Los desarrollos de la medicina hipocrática, 3. El planteo de Aristóteles, entre tradición y fundamentación científica, 4. Las concepciones de Filón y su relación con las teorías griegas, 5. Conclusiones 
Filón perteneció a la comunidad judía de la Alejandría del siglo i y, en este contexto, participó de la educación judeohelenista que combinaba las enseñanzas ancestrales del judaísmo con una formación pedagógica griega en la que se incluían los desarrollos de la literatura, las artes, la ciencia y la filosofia. Sus obras dan cuenta de su búsqueda constante por lograr una síntesis entre sus raíces judías y todo el campo de los saberes griegos, a partir de los cuales llevó a cabo su enorme labor exegética de los textos bíblicos. En general, los estudios que analizan la influencia griega en el pensamiento del filósofo judío se centran en las teorías filosóficas que marcaron en mayor o menor medida sus concepciones teológicas, cosmológicas y antropológicas. No obstante, menor atención han recibido las nociones biológicas, fisiológicas y médicas procedentes de los desarrollos científicos griegos que Filón pudo haber conocido y aceptado, a pesar de que en su obra es posible reconocer una gran cantidad de comentarios o explicaciones que remiten a estos campos del saber. Nuestro trabajo se propone examinar la influencia de esta área del conocimiento griego, la científica y médica, en el pensamiento del filósofo judío, a través del análisis de las teorías que el autor conoce y asume como propias en lo que concierne al proceso de la reproducción sexual. Filón no posee ningún tratado completo dedicado a este asunto, pero en sus escritos se encuentran numerosas afirmaciones aisladas acerca de la gestación, el desarrollo del embrión y la función propia de hombres y mujeres en el proceso reproductivo. Aunque a primera vista parece tratarse de comentarios e ideas desconectadas unas con otras, si se analizan en conjunto permiten reconocer en el pensamiento del alejandrino ciertas concepciones más o menos arraigadas y coherentes entre sí.

Nuestro estudio se centrará en la cuestión de la participación y la función de hombres y mujeres en la gestación e intentará determinar cuáles son los ejes principales de las concepciones filónicas al respecto y cuáles son sus fuentes teóricas o científicas más probables. Con este fin, será necesario rastrear primero las diversas teorías que circulaban en el mundo griego acerca de la reproducción humana y del rol que en ella desempeñan hombres y mujeres. Examinaremos las ideas tradicionales expresadas en la tragedia y provenientes del acervo del pensamiento popular y las confrontaremos con las explicaciones naturales y médicas propuestas por los filósofos presocráticos y por la medicina hipocrática, cuyas teorías se plantearon en directa oposición a las concepciones tradicionales, en especial en lo que respecta a la participación femenina en la gestación. Estudiaremos luego las teorías reproductivas de Aristóteles, filósofo que llevó a su máxima expresión las nociones antiguas y desarrolló el fundamento filosófico y científico para avalarlas. Por último, presentaremos las ideas que Filón expresa sobre este asunto, indagaremos cuál o cuáles de las teorías griegas analizadas ejerció mayor grado de influencia en su pensamiento e intentaremos explicar los motivos por los que Filón se apropia de algunas de estas teorías mientras descarta otras.

\section{DE LA TRAGEDIA A LOS PRIMEROS FILÓSOFOS}

Uno de los temas que más debate ha generado en el mundo griego con respecto a las teorías reproductivas es la pregunta sobre cuál es el aporte femenino a la gestación del embrión, es decir, si la mujer aporta esperma al igual que el hombre o bien funcio- 
na únicamente como receptora del esperma masculino. La respuesta más antigua a este problema se asentó en los roles opuestos que ambos géneros desempeñaban en la organización social, el hombre activo y la mujer pasiva, de modo que relegaba a la mujer a un papel secundario y concedía el protagonismo al varón. En efecto, se consideraba que este aportaba el semen a partir del cual se formaba el embrión, mientras que la mujer proveía únicamente el lugar de la reproducción, actuando como un receptáculo, sin tener incumbencia en la fuerza generativa que impulsaba el movimiento que daba origen y forma al nuevo ser.

Esta concepción del proceso procreativo se expresa ya, aunque desprovista de explicaciones científicas, en los poetas trágicos. Esquilo, en las Euménides, a fin de exculpar el matricidio de Orestes pone en boca de Apolo las siguientes palabras: «No es la llamada madre la que un hijo / engendra, sino nodriza del embrión recién sembrado /

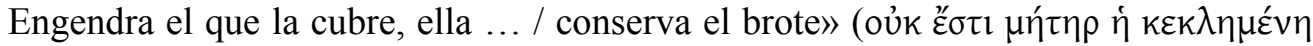

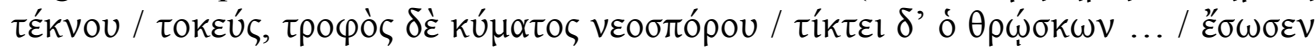
हैpvoc, vv. 658-661) ${ }^{1}$. Idénticas ideas expresa el Orestes de Eurípides: «Mi padre me

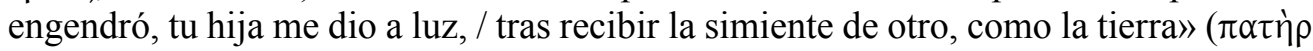

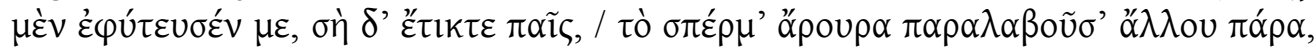
vv. 552-553). Ambos fragmentos trágicos muestran una misma concepción sobre los roles del hombre y la mujer en el proceso procreativo: el padre aporta la semilla

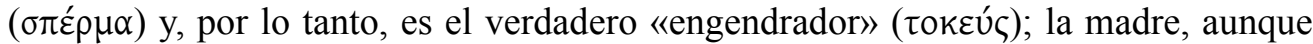

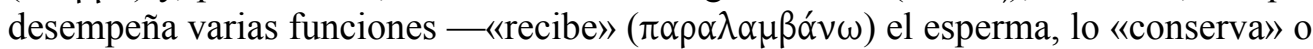

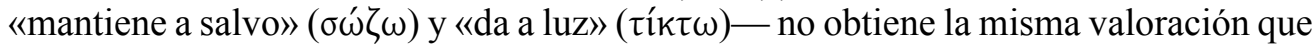

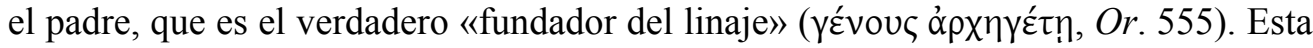
idea, además, se sustenta en una metáfora de antigua tradición que equipara la madre a la tierra de cultivo, donde el hombre siembra la semilla y luego recoge los frutos. $\mathrm{La}$ analogía se expresa en los versos citados a través del abundante vocabulario relativo al

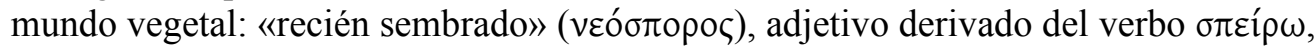

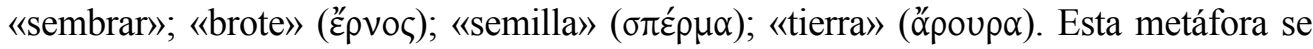
reitera en los poetas griegos desde las obras más tempranas. Por solo citar un ejemplo, Sófocles en Edipo Rey alude a la madre de Edipo como «dos veces campo materno, para

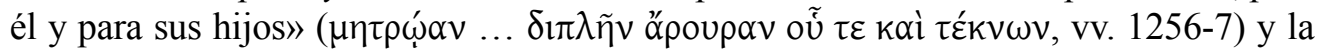

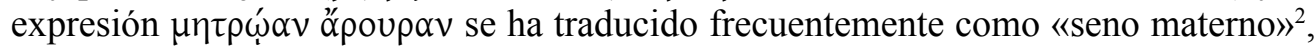
entendiendo el término ópoup $\alpha$ como una metáfora ya lexicalizada para denominar el vientre femenino ${ }^{3}$. Esta vinculación metafórica entre la mujer-madre y el campo de cultivo constituye una forma más de expresión de la concepción de la matriz como el lugar en que el esperma masculino puede desarrollarse; desde esta perspectiva, la mu-

${ }^{1}$ Todas las traducciones de fragmentos de los poetas trágicos, los filósofos presocráticos, el Corpus Hipocrático, Aristóteles y Filón me pertenecen. Las ediciones utilizadas se detallan en el apartado «Ediciones y traducciones». Para las referencias a textos de Filón, empleo las abreviaturas de los títulos latinos establecidas por The Studia Philonica Annual.

${ }^{2}$ Cf. Alamillo 1992; Jebb 1887.

${ }^{3}$ Otros ejemplos en Teognis, Eleg. 1.582; Esquilo, Th. 753-754; Sófocles, Tr. 32; Platón, Ti. 91d; Lg. 839a. 
jer no aporta ningún tipo de materia o sustancia propia al embrión sino que lo recibe como algo ajeno, procedente del exterior ${ }^{4}$.

Con el desarrollo de la filosofía presocrática la discusión acerca del aporte femenino a la gestación se convirtió en uno de los temas centrales en lo relativo a la reproducción. Algunos de los filósofos de la naturaleza adoptaron un enfoque acorde con la teoría tradicional, como atestigua Censorino (De die natali 5.4), quien menciona en este grupo a Diógenes e Hipón y afirma que ambos escribieron que «el embrión se genera a partir del semen del padre solamente» (ex patris tantum modo semine partus nascatur $)^{5}$. No obstante, con la excepción de estos dos autores, puede afirmarse que la teoría preponderante entre los filósofos presocráticos fue la contraria, la tesis de que la madre también aporta sustancia seminal. Los filósofos enumerados por Censorino ${ }^{6}$ (ibid.) como defensores de esta idea son Anaxágoras, Alcmeón, Parménides y Empédocles $^{7}$. A ellos debe sumarse Demócrito, a quien Aecio atribuye la autoría de la teoría según la cual el esperma proviene de todas las partes del cuerpo ${ }^{8}$ y por la refutación de esta idea que realiza Aristóteles - quien argumenta que el hijo no puede tener todas las partes del cuerpo de los dos padres, pues se formarían así dos seres- puede deducirse que ambos progenitores aportan sustancia generativa ${ }^{9}$.

Estas teorías propuestas por los filósofos de la naturaleza implicaban una mirada renovadora que desafiaba los presupuestos tradicionales y permitía aplicar modos de explicación racionales basados en el razonamiento deductivo y la argumentación lógica. A la vez, el estudio biológico del funcionamiento de la reproducción se incluía de esta manera en un sistema mayor de pensamiento en que el universo se entendía como un todo ordenado y regido por leyes que podían descubrirse ${ }^{10}$. En consecuencia, estas primeras indagaciones tuvieron clara influencia en el desarrollo de la medicina hipocrática, que no solo compartió con la filosofía presocrática el enfoque racional y

${ }^{4}$ Cf. García González 2009: 211; Lloyd 1986: 86.

${ }^{5}$ La opinión de Hipón es confirmada por Aecio (= Pseudo-Plutarco), Placita philosophorum 5.5.

${ }^{6}$ Además de los filósofos presocráticos, entre los que adhieren a la primera teoría Censorino incluye a los estoicos, mientras que atribuye a Epicuro la segunda postura.

${ }^{7}$ Cf. también Cens. 6.4-5 sobre Alcmeón y Parménides; Cens. 6.7 y Arist., GA 722b 8 ss. sobre Empédocles; Cens. 6.8 sobre Anaxágoras. Sin embargo, el dato de Censorino sobre este último autor no es certero puesto que Aristóteles lo incluye entre aquellos que afirman que «el esperma procede del macho

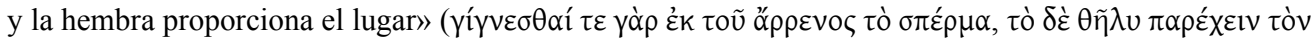

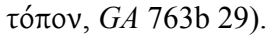

${ }^{8}$ Cf. Aecio, Placit. 5.3, 5.5; Cens. 6.5; Arist., GA 721b 11ss., 722b 6-7 y 764b 11ss. Esta teoría fue denominada modernamente «teoría de la pangénesis» por Erna Lesky (Die Zeugungs- und Vererbungslehren der Antike und ihr Nachwirken, 1951) en su clasificación de las tres teorías existentes en el pensamiento antiguo sobre el origen del esperma: la encéfalo-mielógena, la de la pangénesis y la hematógena (Laín Entralgo 1970: 117). Entre los hipocráticos prevaleció la segunda, aunque hay rastros también de la primera; como veremos más adelante, Aristóteles adoptó la tercera. Cf. también Lloyd (1986: 88) y García González (2009: 214). Sobre la posibilidad de atribución de la teoría de la pangénesis a Anaxágoras además de Demócrito, cf. Lonie 1981: 64-67.

${ }^{9}$ Cf. García González (2009: 208-210) y Lloyd (1986: 87-88). En Longrigg (1993: 54-57) puede consultarse una tabla donde se sintetizan las diversas teorías presocráticas acerca de la reproducción sexual.

${ }^{10}$ Cf. Longrigg 1993: 80-81. 
científico, sino que incluso heredó elementos particulares de sus teorías biológicas y específicamente reproductivas.

\section{LOS DESARROLLOS DE LA MEDICINA HIPOCRÁTICA}

En el ámbito hipocrático prevaleció, al igual que entre los filósofos de la naturaleza, la noción de que ambos progenitores contribuían con sustancia seminal a la procreación ${ }^{11}$. El tratado Sobre la generación es de fundamental relevancia al momento de indagar las concepciones hipocráticas acerca de la gestación y del rol femenino en el proceso. En este escrito se desarrolla la teoría de que el esperma se produce a partir de todas las partes del cuerpo de los dos progenitores, tesis coincidente con la propuesta por Demócrito. El autor hipocrático comienza con una explicación sobre el proceso de formación y el camino recorrido por el semen masculino desde la cabeza hasta los genitales, y luego vuelve la atención hacia la mujer: «La mujer emite tam-

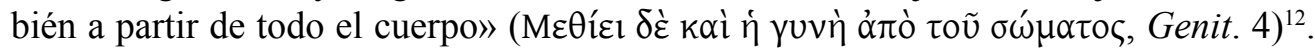
La concepción ocurre inmediatamente después de la unión sexual, si «el esperma de

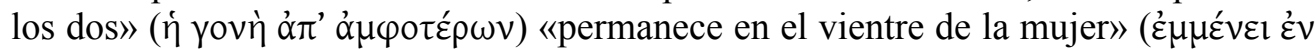

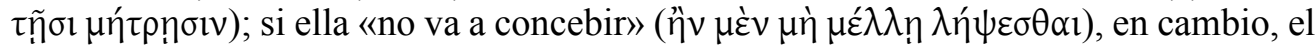

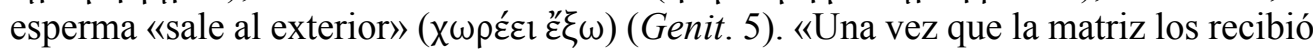

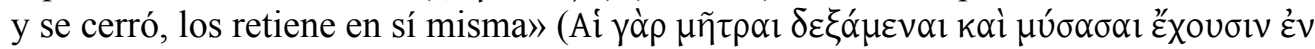
$\dot{\varepsilon} \omega \nu \tau \tilde{n} \sigma \mathrm{lv}$, Genit. 5) y «se mezclan juntos el procedente del hombre y el de la mujer»

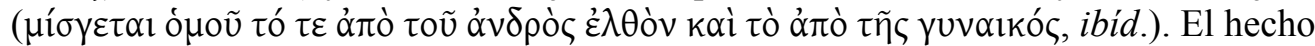
de que la mujer aporte esperma implica que su rol es relevante para diversas cuestiones relacionadas con la constitución del feto, como la determinación del sexo de la criatura según cuál sea la proporción de esperma femenino y masculino aportada por cada progenitor (Genit. 6-7), o la definición de los rasgos fisiológicos del niño, que se asemejará al padre o la madre en aquellas partes desde las que cada uno haya aportado más cantidad de esperma y tendrá siempre similitudes con ambos puesto que «desde

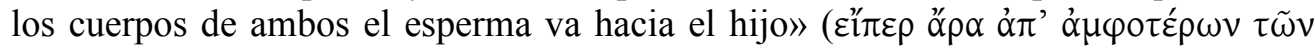

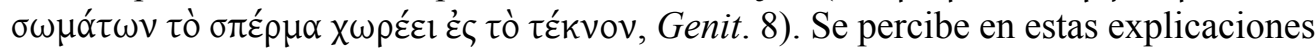
hasta qué punto se considera que la mujer participa al mismo nivel que el hombre en la gestación ${ }^{13}$.

${ }^{11}$ Cf. Laín Entralgo 1970: 116. Longrigg (1993: 63) señala la influencia de Alcmeón y de Demócrito y su teoría de la pangénesis (ibid. 94 ss.) en los hipocráticos, en especial en las teorías reproductivas de Genit., Nat. Puer. y Morb. IV. Sobre la relación entre estos tratados hipocráticos en particular y la filosofía presocrática, cf. Lonie 1981: 62-70 y Rodríguez Blanco 2003: 244.

${ }^{12}$ Cito la edición de los textos hipocráticos de Littré (1961-1962), aunque he cotejado también las ediciones de Loeb (Jones 1959, Potter 1995 y 2012) y del Corpus Medicorum Graecorum (Grensemann 1968, Joly 2003). Cuando existen diferencias significativas entre las ediciones, las señalo en nota.

${ }^{13}$ Para una exposición sobre la embriología propuesta en Genit. y Nat. Puer. y sobre la forma en que estos tratados explican la definición del sexo del embrión y la herencia de los caracteres somáticos, cf. Laín Entralgo 1970: 120-124 y Lonie 1981: 119-139, quien ofrece el comentario detallado de los pasajes que hemos citado. 
También en los dos restantes tratados que forman un conjunto con Sobre la generación —Sobre la naturaleza del niño y Enfermedades $I V^{14}$ — se reitera la misma idea acerca de la contribución femenina a la generación: cuando la matriz retiene el esperma de hombre y mujer ambos se mezclan y se concibe el embrión (Nat. Puer. 12; Morb. 4.32 ${ }^{15}$ ). Asimismo, otros escritos del Corpus Hippocraticum coinciden en esta noción. En Sobre las enfermedades de las mujeres se afirma que si el cuerpo de la mujer se encuentra en malas condiciones de salud y tiene menstruaciones biliosas, «se debilita la simiente de ambos, la del hombre y la de la mujer, y no permanece en el

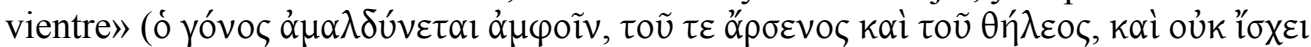

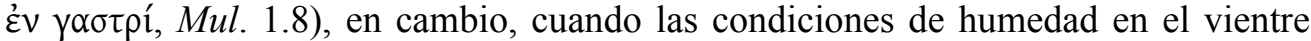
femenino son las adecuadas, «si [el semen] que sale del hombre confluye con el pro-

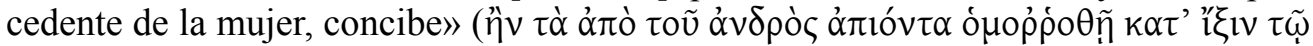

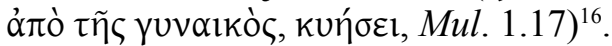

En conclusión, las teorías hipocráticas, en línea con las ideas prevalecientes en la filosofia presocrática, desarrollan una nueva concepción del proceso de gestación, en la que las contribuciones de los dos progenitores - hombre y mujer - son equivalentes y en la que tanto el aporte de uno como de otra puede ser predominante, lo que influirá, por ejemplo, en la definición del sexo del embrión o en las semejanzas con su padre o madre. Si bien estas ideas se encontraban ya en ciernes en las propuestas de los filósofos de la naturaleza, la medicina despliega un aparato de argumentación lógica basado en la observación empírica con el fin de asentar la racionalidad y justificación de esta teoría, que resultaba revolucionaria respecto de las nociones tradicionales cimentadas en el papel subordinado y pasivo que la mujer desempeñaba en la vida socia ${ }^{17}$. Sin embargo, estas voces disonantes con respecto a la ideología prevaleciente conviven con otras que realzan la validez de las concepciones asentadas en la tradición popular e intentan justificarlas científicamente.

${ }^{14}$ Los tres tratados se han editado en conjunto pues Littré consideró que podían formar parte de una única obra. Hay desacuerdos respecto de esta teoría, en especial acerca de la inclusión del tercero de los títulos en un tratado único; cf. las objeciones de Lonie 1981: 43-45 y la síntesis de Rodríguez Blanco 2003: 233-238. Sin embargo, parece muy probable la misma autoría para los tres escritos (cf. Lonie 1981: 46-51; Rodríguez Blanco 2003: 239-240). La datación de los textos puede ubicarse entre fines del siglo v y principios del IV a.C. (cf. Rodríguez Blanco 2003: 243-245 y Lonie 1981: 71, que establece la fecha alrededor del año 420 a.C.).

${ }^{15}=$ Nat. Puer. 1 y Morb. 4.1, ed. Potter. Cf. el comentario a estos pasajes en Lonie 1981: 146-158 y 257-259.

${ }^{16}$ Cf. también Vict. 25-27.

${ }^{17}$ Lloyd (1986: 94) enfatiza la importancia de estas ideas renovadoras, aunque aclara que su alcance es limitado. Así, con respecto a los tratados Sobre la generación y Sobre la dieta, afirma: «they represent [...] important dissenting voices against the notion of the essential disparity between the contributions of the two parents. This is certainly not to challenge the whole prevailing ideology in relation to the female sex: yet it does provide alternatives to one type of biological theory that —of set purpose or otherwiseunderpinned that ideology». 


\section{EL PLANTEO DE ARISTÓTELES, ENTRE TRADICIÓN Y FUNDAMENTACIÓN CIENTÍFICA}

Frente a las innovadoras propuestas de los presocráticos y de la medicina hipocrática, Aristóteles despliega una teoría contraria que vuelve sobre las viejas concepciones pero las asienta sobre una argumentación lógica, cuidadosamente razonada y basada en la exploración científica. El tratado que mayor información provee acerca de sus teorías reproductivas y embriológicas es Sobre la generación de los animales, donde el filósofo, además de exponer sus propias teorías acerca del funcionamiento de la reproducción sexual, discute y refuta aquellas ideas precedentes que considera incorrectas, entre otras, la teoría de la pangénesis ${ }^{18}$ y de que tanto el hombre como la mujer aportan esperma en el proceso de gestación.

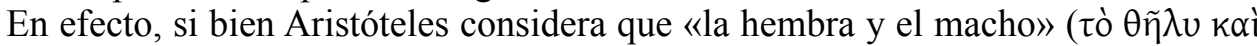

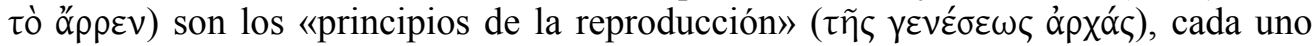
constituye un principio de distinta calidad, «el macho como poseedor del principio

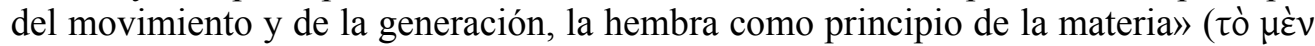

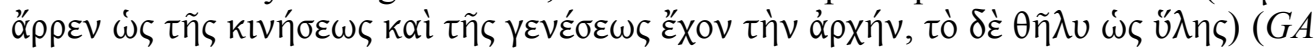
716a 2-7) ${ }^{19}$. Esta diferencia entre los roles femenino y masculino se sustenta en los aportes disímiles que cada uno puede realizar. Según el estagirita, el esperma es «un

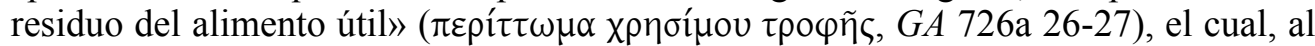
ingresar al organismo, es sometido a un proceso de cocción gracias al calor corporal de los seres vivos y de ese modo se transforma en sangre. Los hombres, además, someten a parte de esa sangre a una cocción más acabada que la convierte en esperma (GA 726b 1-12), pero la hembra, que es más fría, no puede efectuar este segundo nivel de cocción, de modo que el residuo de los alimentos convertidos en sangre constituye la sustancia de las menstruaciones (GA 726b 30-727a 1; 728a 18-21). Por lo tanto, para Aristóteles «es evidente que la hembra no contribuye con esperma a la generación»

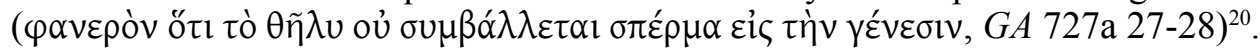

Sin embargo, esta definición de la naturaleza y origen del esperma y de las menstruaciones no acaba de aclarar por qué motivo el hombre es principio generativo y la mujer principio material. La explicación de esta diferencia radica en las distintas funciones que estos dos materiales son capaces de desempeñar en el proceso reproductivo. Las menstruaciones, en tanto no tienen la fuerza dinámica y generativa del esperma, constituyen la materia a partir de la cual se formará el embrión. En cambio, la materia del semen no contribuye a la gestación ni se mezcla con la materia femenina, sino que este actúa como proveedor de «la potencia y el movimiento que hay

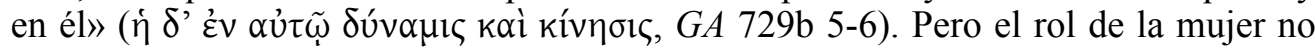

${ }^{18}$ Cf. $G A 721 \mathrm{~b}$ ss.

${ }^{19}$ Como puede apreciarse en estas primeras definiciones, el filósofo analiza el funcionamiento de la reproducción sexual tomando como punto de partida ciertos conceptos básicos de su pensamiento metafísico, tales como las nociones de materia-forma, potencia-acto y las causas que rigen todo proceso: formal, material, eficiente y final. Cf. Sánchez 1994: 30 y García González 2009: 212.

${ }^{20}$ En Historia de los animales 636a-637b Aristóteles parece asumir la teoría de los dos espermas, masculino y femenino, aunque, a diferencia de la cuidadosa exposición aquí analizada, no explica ni justifica esta postura. 
acaba con la contribución de la materia; además, la hembra proporciona el lugar de

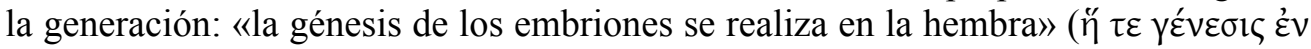

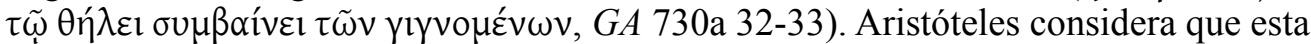
función femenina es natural, puesto que si ella es la que provee la materia, es lógico que allí mismo debe producirse la formación del feto, de igual modo que el carpintero trabaja donde se halla la madera y el alfarero donde está el barro. De esta manera, el feto se formará a partir de toda la materia que necesite y la hembra seguirá aportando más cantidad de materia a medida que avanza el crecimiento (GA 730b 1-9).

Como señala Lloyd (1986: 98), a pesar de que a lo largo de su trabajo Aristóteles da muestras de utilizar métodos de experimentación científica para justificar sus afirmaciones, esta teoría acerca de las funciones del esperma y la menstruación parece basarse en gran medida en sus preconcepciones con respecto a los roles sociales de hombres y mujeres y a la subordinación y pasividad de la mujer ${ }^{21}$. Ello se aprecia en las explicaciones de la teoría que acabamos de exponer que reitera en el capítulo 729a-b: «Si el

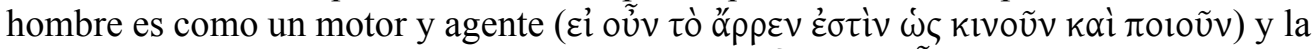

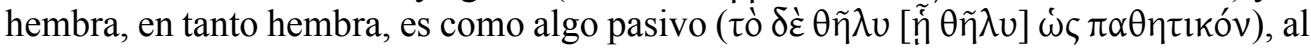
semen del macho la hembra no aportaría semen, sino materia» (GA 729a 28-31); «la hembra, en cuanto hembra, es pasiva, y el macho, en cuanto macho, es activo y por

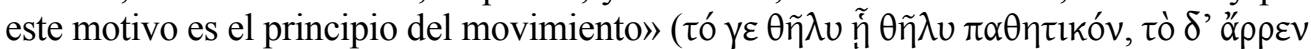

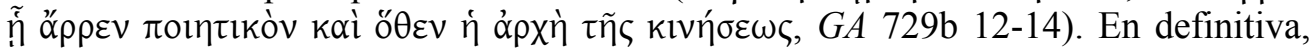
Aristóteles, retomando las teorías tradicionales basadas en los roles disímiles atribuidos a hombres y mujeres en la vida social, niega la aportación de simiente generativa por parte de la hembra. Esta contribuye únicamente con elementos pasivos a la gestación: la materia y el lugar de la generación. El hombre, en cambio, provee el principio motor y dinámico que da forma a la materia femenina, movimiento sin el cual no sería posible la concepción y el desarrollo del nuevo ser. El filósofo se distancia así enormemente de la teoría hipocrática y de las concepciones presocráticas que habían asignado a hombres y mujeres un rol equivalente en el proceso reproductivo.

\section{LAS CONCEPCIONES DE FILÓN Y SU RELACIÓN CON LAS TEORÍAS GRIEGAS}

Como hemos adelantado en la introducción de este trabajo, Filón no ha redactado un tratado completo dedicado a las cuestiones reproductivas y embriológicas al modo de los escritos hipocráticos y aristotélicos analizados. Sin embargo, las numerosas referencias a los procesos generativos que pueden hallarse a lo largo de sus obras muestran una concepción bien definida y arraigada en su pensamiento. Para empezar, una primera afirmación al respecto se encuentra en el tratado La creación del mundo según Moisés (Opif.), escrito que funciona como inicio o apertura de las dos series más importantes de

${ }^{21}$ «Although Aristotle has some powerful arguments against the theory that the seed is drawn from the whole of the body, he appeals to a number of patently a priori considerations to support his own thesis. To define the female in terms of an incapacity is to assume that the male provides the model and the norm» (Lloyd 1986: 98). 
la exégesis filónica, el Comentario Alegórico y la Exposición de la Ley ${ }^{22}$. Allí el filósofo, al analizar el orden necesario que rige la creación, señala: «sucede que el esperma es

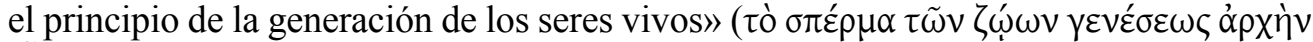

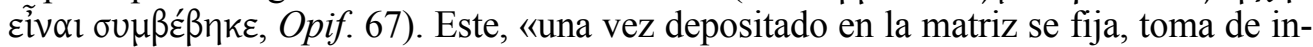

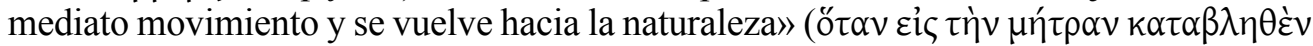

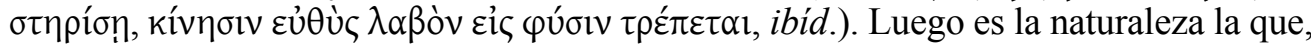
al modo de un excelente artista, «plasma al ser viviente» ( $\zeta \omega 0 \pi \lambda \alpha \sigma \tau \varepsilon \tilde{\imath})$. En estas palabras filónicas pueden reconocerse ciertos puntos de contacto con la teoría desarrollada por Aristóteles, en la que el esperma era uno de los «principios de la generación»

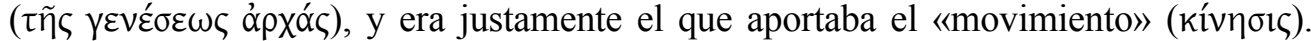

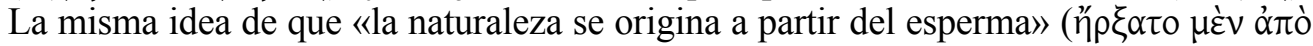

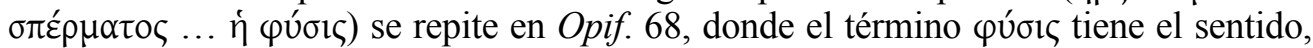
más que de 'naturaleza', de 'origen', 'nacimiento', 'crecimiento', es decir, alude a la fuerza generativa de la naturaleza. Y a esta idea se añade la noción del movimiento en Alegoría de las leyes (Leg.) 2.37, donde se afirma que «la generación es producida por

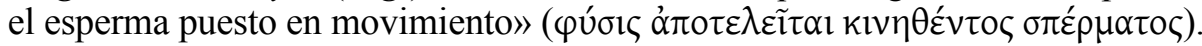

En estas primeras aproximaciones no puede determinarse si Filón entiende que el esperma proviene únicamente del hombre o es producido también por la mujer, puesto que en uno $\mathrm{u}$ otro caso igualmente necesitará fijarse en el vientre femenino para desarrollarse, como asienta en Opif. 67. No obstante, en otros pasajes resulta claro que la concepción filónica asigna a hombres y mujeres roles muy disímiles en el proceso generativo. Así, en Sobre Abraham 101 distingue sus dos funciones básicas: «en el matri-

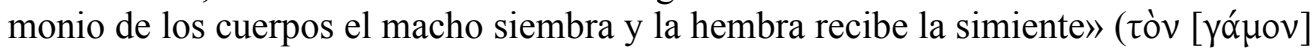

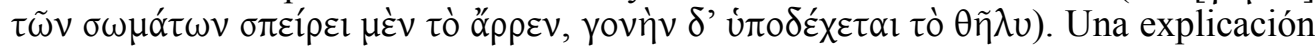
más pormenorizada ofrece Filón en Sobre la indestructibilidad del mundo (Aet.). Como el nombre de este tratado indica, la temática de la que se ocupa es la eternidad del cosmos y de las especies que hay en él, contexto en el que el alejandrino refuta las teorías que proponen la destrucción y recreación cíclica del cosmos. En este marco Filón aclara que los humanos no poseen la inmortalidad individualmente pero sí como especie, lo que brinda la ocasión para explicar cómo funciona el proceso a través del cual «los seres humanos brotan de los seres humanos» ( $\xi^{\prime} \xi \alpha v \theta \rho \omega \tilde{\omega} \omega \nu \nu \beta \lambda \alpha \sigma \tau \alpha \dot{v} v o v \sigma v v$

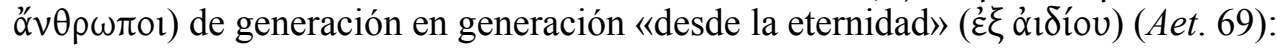

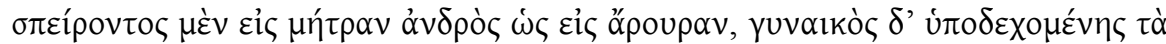

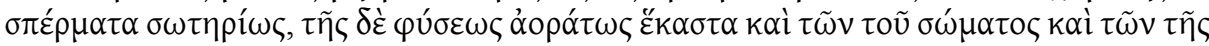
$\psi \cup \times \tilde{n} \varsigma \delta 1 \alpha \pi \lambda \alpha \tau \tau o u ́ \sigma n \varsigma ~ \mu \varepsilon \rho \tilde{\omega} v$

el hombre es quien siembra en la matriz como en una tierra de cultivo, la mujer quien recibe las simientes y las mantiene a salvo, y la naturaleza es la que moldea invisiblemente cada una de las partes, tanto las del cuerpo como las del alma (Aet. 69).

${ }^{22}$ El Comentario está compuesto por veinte tratados que aplican la exégesis alegórica a una lectura continua del Génesis; la Exposición ofrece una lectura sistemática de los libros del Pentateuco, que se ocupa primero de las vidas de los Patriarcas como modelos de las leyes no escritas y luego de la Ley mosaica organizada bajo el esquema clasificatorio del Decálogo. Cf. Martín 2009: 28-36. Sobre la ubicación de La creación del mundo según Moisés en relación con estas series, cf. Martín 2009: 25. 
Estos pasajes evidencian una concepción del proceso procreativo en que las funciones femeninas y masculinas son claramente dispares: el hombre es quien aporta

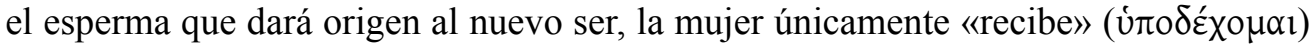
esa simiente y la conserva «a salvo» ( $\sigma \omega \tau \eta p i ́ \omega \varsigma)$ en su interior mientras se produce su maduración. Además de encontrarse mucho más cercana a la teoría aristotélica que a la hipocrática, en esta descripción del proceso los términos que hemos destacado muestran estrechas similitudes con las concepciones que analizamos en la poesía trágica, donde las acciones de la mujer en la procreación también consistían en «recibir»

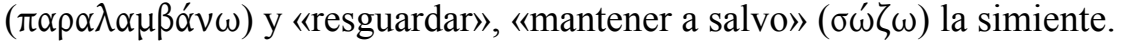

Pero además, en el párrafo citado Filón se apropia de la imagen más característica de la concepción tradicional de la procreación, la metáfora que vincula el vientre femenino con la tierra de cultivo (ópoup $\alpha$ ) y la tarea del hombre con la de la siembra

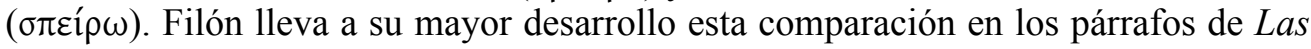
leyes particulares (Spec.) 3 en que explica la prohibición bíblica de ciertas relaciones sexuales que necesariamente resultarán infecundas. Así, en el caso de un hombre que mantenga relaciones con una mujer durante el período de la menstruación en contra

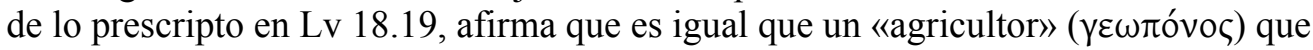
«sembrara los granos de trigo o cebada en pantanos y torrentes en lugar de las llanu-

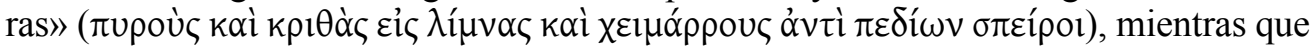
«para obtener abundancia de frutos es necesario esparcir la semilla en los campos

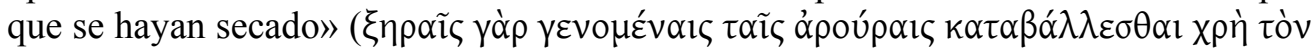

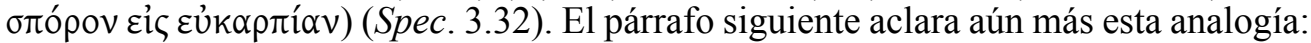
«la naturaleza», afirma Filón, «purifica cada mes el vientre como una admirable tierra

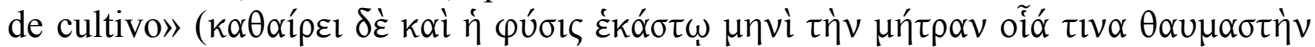

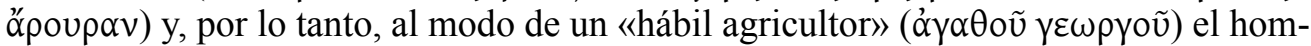

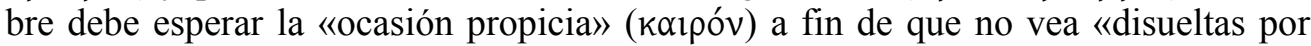
completo» (

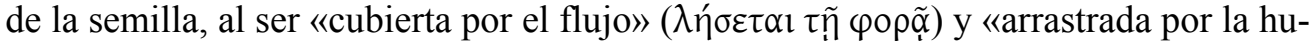

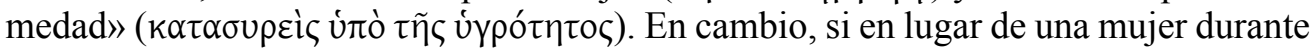
su menstruación se tratara de una mujer estéril, la interdicción de las relaciones se justifica no por el exceso de humedad sino de su contrario, la sequedad, de modo que

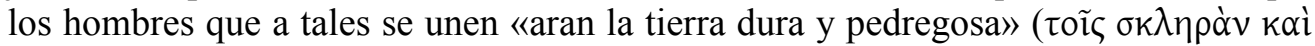
$\lambda_{\imath} \theta \omega \dot{\delta} \eta \eta \tilde{\eta} v \alpha \dot{\alpha} \rho o \tilde{\sigma} \sigma \mathrm{lv}$, Spec. 3.34) ${ }^{23}$.

Estas explicaciones que ofrece Filón, a pesar de su carácter metafórico, expresan nuevamente una concepción del proceso reproductivo en que el hombre tiene un papel activo, pues provee el esperma con su fuerza generativa, mientras que la mujer lo recibe en forma pasiva y solo si su vientre se encuentra en las condiciones adecuadas podrá conservar la simiente y resguardarla durante su crecimiento. La noción tradicional ya manifiesta en la tragedia ática, retomada solo por algunos de los filósofos pre-

${ }^{23}$ Por último, la analogía se aplica a las relaciones entre hombres, pues quien participa de estas actúa

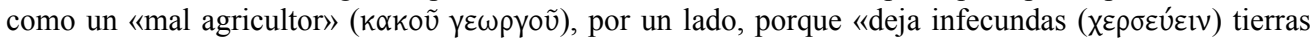

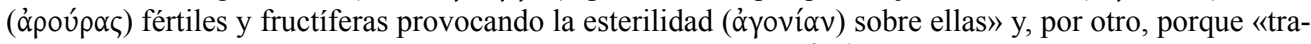

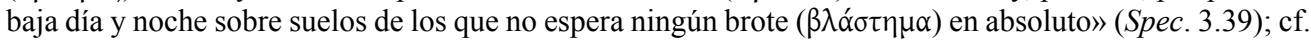
Contempl. 62. 
socráticos y luego llevada a su máximo desarrollo por Aristóteles, es la que prevalece en el pensamiento del alejandrino.

Sin embargo, la preferencia por la concepción más tradicional no implica el desconocimiento de las teorías científicas existentes al respecto. En efecto, a pesar de la imaginería agrícola que utiliza, cuando se refiere a la menstruación el filósofo desarrolla ideas coincidentes con las sostenidas en el ambiente de la medicina hipocrática y también asumidas por Aristóteles. En el tratado hipocrático Enfermedades de las mujeres 1.17 se manifiesta igualmente la preocupación por evitar los excesos de humedad

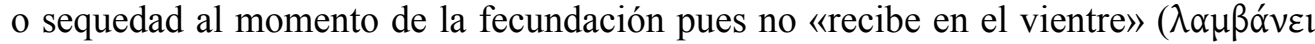
$\delta \dot{\varepsilon}$ ćv $\gamma \alpha \sigma \tau \rho i ́)$ es decir, no quedará embarazada, «ni la [mujer] que tiene humedad, ni la

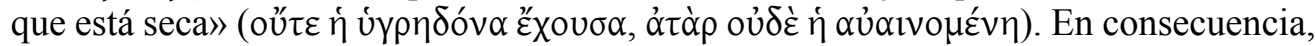
el mejor momento para la concepción es «cuando terminan o cuando comienzan las

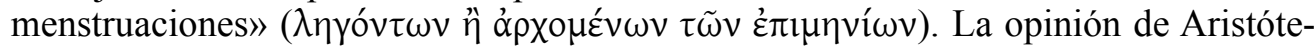
les es coincidente en este aspecto, pues asegura que «la hembra no concebiría si las menstruaciones no se producen en absoluto ni tampoco cuando, estando presentes, se-

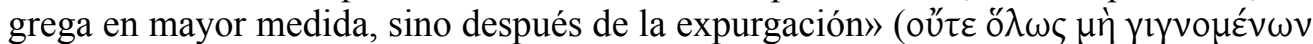

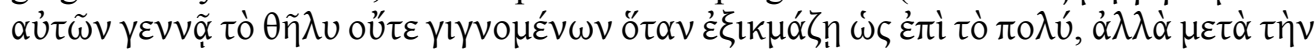

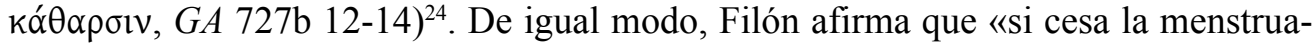

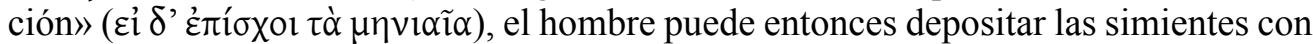
confianza sin temer su destrucción (Spec. 3.33).

Además de su importancia para determinar el momento apto para la concepción, la menstruación adquiere otra función en la teoría reproductiva asumida por Filón. En efecto, si bien la mujer no aporta esperma, sí debe proveer al embrión de alimentación $^{25}$, humedad y de la materia que necesita para formarse. Esta materia para Filón, al igual que para Aristóteles, la suministran los flujos menstruales. En efecto, en el tratado La creación del mundo según Moisés, en el contexto de su interpretación de la fuente del paraíso (Gn 2.6) el filósofo judío desarrolla los argumentos que demuestran la importancia primordial del agua para la vida. Dado que la generación de ninguno de los seres vivos podría producirse sin humedad, resulta necesariamente que la mencionada sustancia húmeda es parte de la tierra engendradora de todas las cosas, «al

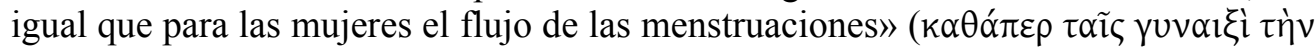

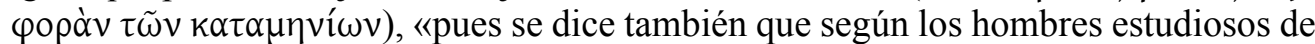

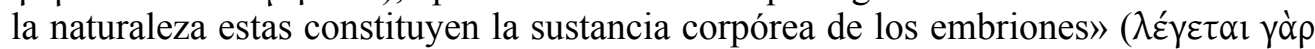

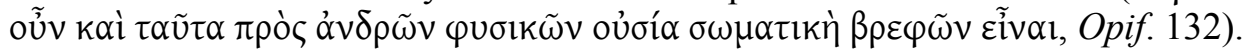

En definitiva, el análisis desarrollado hasta aquí demuestra cabalmente que Filón asumió como propia la noción tradicional de la reproducción que otorgaba a hombres y mujeres roles claramente diferenciados y a los que se atribuía diverso grado de importancia. Esta concepción incorpora las imágenes arcaicas difundidas en la literatura griega más antigua que ubican al hombre en el papel activo de la siembra y a la mujer

${ }^{24}$ Véase la misma idea en Arist., HA 582b 11-12.

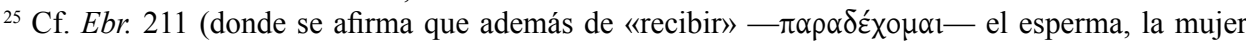
debe «criar»o «nutrir, alimentar» —á $v \alpha \tau \rho \varepsilon ́ \varphi \omega —$ lo que ha sido depositado en ella) y Aet. 98 (donde se destaca la importancia de la «nutrición»o «alimento» - $\tau \rho \circ \varphi$ - que suministra la madre para la supervivencia del embrión). 
en el rol pasivo de la tierra que recibe la simiente fecundante. A la vez, Filón adhiere a las nociones defendidas por Aristóteles, quien atribuye a los roles disímiles de hombre y mujer un orden jerárquico: la más relevante es la función del varón que aporta movimiento y es por tanto agente; en un lugar inferior se ubica la materia pasiva e inerte. Un párrafo de Cuestiones sobre el Génesis $(Q G)$ ofrece la formulación más explícita de estas ideas en el pensamiento de Filón: «la materia de la hembra en los restos de los fluidos menstruales produce el feto, pero el macho provee el arte y la causa» y por lo tanto «el hombre provee la parte mayor y más necesaria en el proceso de la generación» $(Q G 3.47)^{26}$.

No obstante, la adhesión a esta teoría por parte del filósofo alejandrino plantea la pregunta acerca de sus motivaciones, puesto que es muy difícil suponer un total desconocimiento de las teorías hipocráticas, que eran las más aceptadas en el contexto científico y médico de su época ${ }^{27}$. De hecho, varios autores han señalado que Filón poseía conocimientos médicos bastante amplios, de los cuales se encuentran numerosos rastros a lo largo de su obra. Hogan (1992: 192), en este sentido, ha comprobado que en los escritos de Filón pueden hallarse precisas observaciones sobre procedimientos médicos, muchos términos provenientes del lenguaje técnico de la medicina, así como referencias a Hipócrates y a las escuelas médicas, lo que demuestra para este estudioso que Filón poseía «a medical background» ${ }^{28}$. Igualmente Dorothy Sly (1996: 156) considera que las obras de Filón revelan un amplio conocimiento de la práctica médica e incluso supone que entre las lecturas de Filón se incluirían los escritos hipocráticos. En lo que respecta específicamente al funcionamiento de la reproducción, pueden hallarse en la obra filónica muchas afirmaciones que remiten claramente a las concepciones hipocráticas, en especial, a las teorías embriológicas sobre el desarrollo del feto en el vientre materno, los tiempos que este suponía y los períodos adecuados para un parto saludable ${ }^{29}$.

De hecho, Filón cita directamente al médico griego acerca del tiempo que requiere la concepción del embrión: «Dice Hipócrates, el conocedor de la naturaleza, que en siete días se consolida tanto la fijación del semen como la formación de la carne»

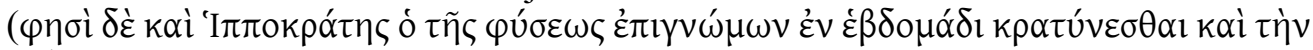

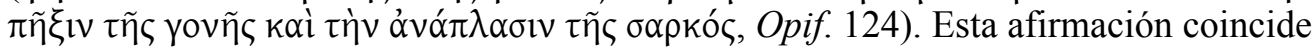

${ }^{26}$ Dado que los tratados Cuestiones sobre el Génesis 1-4 no se han conservado en griego (con la excepción de algunos fragmentos) sino en una traducción armenia, no puedo incluir aquí la cita en idioma original y traduzco a partir de la edición inglesa de Marcus (1953: 242): «the matter of the female in the remains of the menstrual fluids produces the fetus. But the male (provides) the skill and the cause [...] the male provides the greater and more necessary (part) in the process of generation». El traductor

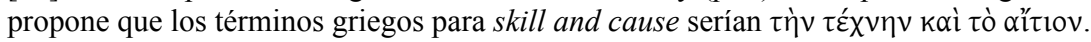

${ }^{27}$ Cf. Sly (1996: 155): «Medicine in Philo's Alexandria professed to follow the principles laid down by the legendary physician Hippocrates [...] No figure matched his degree of influence in the field until Galen, who lived in the second century CE».

${ }^{28} \mathrm{El}$ autor realiza un análisis de los elementos hipocráticos que aparecen en la obra de Filón, cf. Hogan 1992: 192-207. También Bréhier (1950: 286), importante estudioso de la obra filoniana, afirma: «Il est sûr en tout cas que Philon reçut une éducation médicale; il a connu quelques fragments de la collection hippocratique».

${ }^{29}$ Cf. Sly (1996: 160): «Philo did enjoy theoretical discussions among natural philosophers and physicians about the development of the foetus». 
cabalmente con las expuestas en algunos tratados del Corpus Hipocrático. En Sobre las carnes 19 se asienta que los períodos en el ser humano son de siete días y que el primero sucede «cuando el semen llega a la matriz, en siete días tiene todo cuanto debe

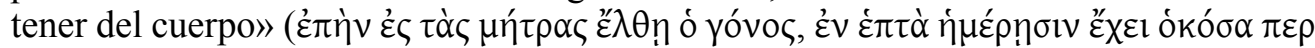

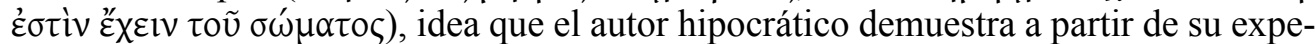
rimentación empírica con fetos abortados por prostitutas ${ }^{30}$.

Otra teoría que Filón tomó de los hipocráticos es la que supone que los embriones nacidos en partos de ocho meses de gestación no pueden vivir, mientras que los de siete o nueve meses sí sobreviven. Esta idea se reitera en La creación del mundo según Moisés 124 y en Alegorías de las leyes 1.9, en ambos casos al modo de una verdad indiscutible: «los que nacen a los siete meses viven, mientras que los de ocho meses en

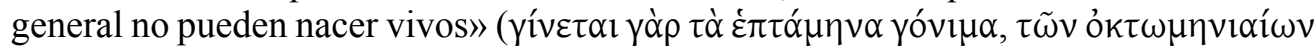

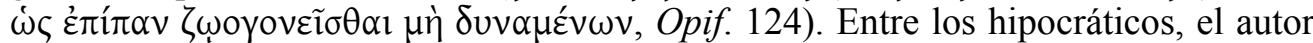
de Sobre el parto de ocho meses asienta en forma categórica que «los nacidos de ocho

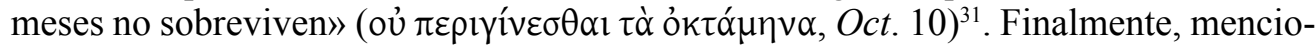
naremos una última idea en que las concepciones de Filón y los hipocráticos resultan cercanas, la que propone que los embriones varones tardan menos tiempo en formarse en todas sus partes que los de las hembras. En varios pasajes de Cuestiones sobre el Génesis Filón reitera que el varón tarda cuarenta días en formarse por completo, mientras que la hembra requiere el doble de tiempo, ochenta días $(Q G 1.25,2.14,4.27)$. De igual modo, en el escrito hipocrático Sobre el parto de siete meses $9^{32}$ se afirma que los primeros cuarenta días de gestación son decisivos porque es cuando se modelan todas las partes del embrión, pero mientras los varones están completamente formados en ese tiempo, en el caso de las hembras lleva más tiempo que se distingan sus partes. Sobre la naturaleza del niño $18^{33}$ presenta una teoría similar, aunque los períodos temporales son distintos de los mencionados por Filón: el niño tarda treinta días en formarse y la niña cuarenta y dos. Pero a pesar de estas diferencias en cuanto a los períodos concretos, entre los hipocráticos parece bien asentada la idea de que el embrión varón se forma más rápido que la hembra ${ }^{34}$.

${ }^{30}$ La misma aseveración respecto de los primeros siete días del embrión aparece en Sobre las semanas (o Sobre la hebdómada) 1 y 10, un tratado que ha ejercido gran influencia en Filón (cf. Hogan 1992: 192-194 y Bréhier 1950: 286). Filón también cita las siete etapas de la vida humana (Opif. 105) y las siete secreciones del cuerpo humano (Opif. 123, Leg. 1.13), atribuyéndolas explícitamente a Hipócrates.

${ }^{31}=$ Oct. 2, ed. Grensemann. Cf. también Sept. 3, 5, 6, etc. (= Oct. 3, 5, 6, ed. Potter; Oct. 6, 9, 10, ed. Grensemann. La diferencia de títulos se debe a que Littré divide en dos tratados contiguos - Sobre el parto de siete meses 1-9; Sobre el parto de ocho meses 10-13 - lo que otros editores consideran un único tratado Sobre el parto de ocho meses. Las discrepancias en la numeración de capítulos surgen por las dificultades de estructura que ofrece el tratado, motivadas por diferentes tradiciones manuscritas y por problemas de estructura interna; al respecto cf. Grensemann 1968: 41-47; Jouanna 1973; Joly 1976; Villa Polo 2003: 346-353). Aristóteles es más laxo en esta temática y afirma que los bebés de ocho meses viven, aunque en menor proporción, cf. GA 772 b 10.

${ }^{32}=$ Oct. 9, ed. Potter; Oct. 1, ed. Grensemann.

${ }^{33}=$ Nat. Puer. 7 , ed. Potter.

${ }^{34}$ Aristóteles también comparte esta concepción (GA 775a), aunque los períodos de tiempo que define para la formación de niños y niñas son diferentes: cuarenta días para el varón y noventa para la hembra (cf. $H A$ 583b). 
Las coincidencias que hemos reconocido hasta aquí entre nociones expresadas por Filón y las teorías embriológicas hipocráticas demuestran que Filón contaba con conocimientos de las ideas que circulaban en los ámbitos médicos, e incluso aceptaba muchas de las nociones desarrolladas entre los hipocráticos. En consecuencia, su apropiación de la teoría aristotélica que propone una disímil participación de hombres y mujeres en el proceso generativo no está motivada por el desconocimiento de otras concepciones al respecto, sino que se trata de una elección ideológica consciente. Y si ubicamos las ideas reproductivas de Filón en el contexto general de su pensamiento filosófico, y especialmente antropológico, podremos reconocer de inmediato la coherencia que guardan entre sí.

En efecto, así como antes afirmamos que Aristóteles, a pesar de los argumentos racionales y científicos en que sustenta su teoría, parte de consideraciones apriorísticas acerca de la jerarquía entre hombres y mujeres y los roles sociales de cada uno, en Filón podemos identificar un tratamiento similar del problema ${ }^{35}$. Como demuestra Mattila (1996), el filósofo judío desarrolla un sistema clasificatorio del cosmos organizado en categorías que forman pares binarios de opuestos y se ubican en una jerarquía. En ese sistema, masculino y femenino constituyen dos categorías opuestas que se ponen en correlación con todos los demás pares jerárquicos de opuestos, de modo que lo 'masculino' se identifica con lo racional, lo ideal e incorpóreo, lo celestial, indivisible e invariable, y con el principio activo. Lo 'femenino', en cambio, se identifica con todas las categorías que, opuestas a las anteriores, son también de naturaleza inferior: lo irracional, lo perceptible y material, lo terrenal, divisible y cambiante, el principio pasivo $^{36}$. En esta clasificación jerárquica resulta sumamente justificado considerar que el hombre desempeña un papel activo y generativo en tanto que la mujer ocupa un rol pasivo y solo aporta un elemento material, carente de fuerza y dinamismo.

Uno de los lugares en que esta concepción se expresa en forma explícita resulta significativo, pues Filón distingue los roles activo y pasivo de hombre y mujer justo después de haber mencionado, como ejemplo de actividad, que «la generación es producida por el esperma puesto en movimiento» (Leg. 2.37, citado supra). Esta aserción se produce en el contexto de su interpretación de la creación de la mujer, en la que Filón explica que el hombre — Adán_ representa el intelecto y la mujer recién creada simboliza la sensibilidad que funcionará como ayudante del intelecto; en este marco, afirma: «así como el varón se manifiesta en el actuar y la mujer en el padecer, de igual modo el intelecto se encuentra en la actividad, y la sensibilidad, al modo de una mujer,

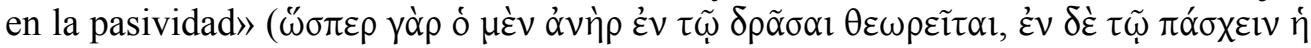

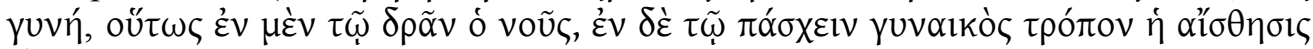

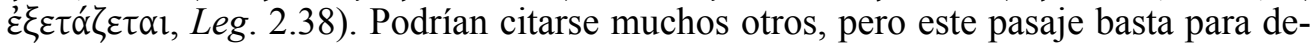
mostrar que Filón distingue los roles de hombre y mujer en términos de actividad y pasividad, al igual que sucedía en el pensamiento de Aristóteles. Es esta ideología la que conduce al alejandrino a adoptar la teoría que diferencia, en el proceso generativo, las mismas dos funciones para hombres y mujeres, el hombre aporta el principio del

${ }^{35}$ Cf. Sly 1996: 161.

${ }^{36}$ Mattila 1996: 106. Sobre la oposición actividad-pasividad en relación a las categorías de lo masculino y lo femenino, cf. también Baer 1970: 62-63. 
movimiento, mientras la mujer pasivamente recibe ese principio generativo que dará forma a la materia inerte que ella provee. Más allá de que Filón pudiera conocer los lineamientos principales de las teorías hipocráticas y sus antecedentes en la filosofía presocrática, la teoría más tradicional y su fundamentación aristotélica guardan mayor coherencia con su propio sistema filosófico y antropológico.

\section{CONCLUSIONES}

El análisis desarrollado a lo largo de nuestro trabajo ha permitido demostrar que Filón conocía muchas de las teorías fisiológicas y médicas acerca de la reproducción sexual que circulaban en su entorno helenístico y que hundían sus raíces en los primeros desarrollos filosóficos de los presocráticos, la medicina hipocrática y los estudios biológicos y naturales de Aristóteles. A pesar de que el filósofo judío no dedica ningún tratado completo a las temáticas relacionadas con la reproducción y la embriología, claramente posee ciertas concepciones definidas y coherentes que se manifiestan en comentarios o breves explicaciones formuladas de manera similar o coincidente en diversos puntos de sus escritos. Estas referencias muestran una comprensión de la reproducción sexual anclada en las teorías más tradicionales del mundo griego, que se manifestaban en la imaginería que ligaba la mujer con la tierra y la función fecundadora del hombre con la siembra. Esta noción antigua de la procreación es fundamentada científicamente por Aristóteles, quien identifica los papeles de hombre y mujer con los roles de actividad y pasividad, forma y materia, y los ubica en una gradación en la que los primeros son superiores a los segundos. Así, la función activa del hombre que provee la sustancia seminal como principio del movimiento y de la forma es superior y por lo tanto más importante que el papel pasivo femenino de recepción del principio activo y aporte de la materia informe. Filón retoma estos mismos conceptos porque su filosofía ubica de la misma manera las jerarquías entre 'masculino-femenino', 'activo-pasivo' y 'forma-materia', de modo que la teoría tradicional cimentada mediante métodos racionales y lógicos por Aristóteles es la que mejor se acomoda a su propia orientación filosófica e ideológica.

Ello no implica, no obstante, postular que Filón desconocía las teorías hipocráticas y de la mayoría de los presocráticos, que proponían una visión renovadora en la que el papel de los sexos se volvía equivalente y la mujer aportaba sustancia espermática al igual que el hombre. De hecho, las numerosas referencias a otros aspectos de la teoría embriológica hipocrática permiten suponer que Filón habría conocido al menos en sus líneas generales la teoría médica que mayor aceptación poseía en su contexto histórico-cultural. Sin embargo, el alejandrino no se refiere a estas teorías porque no le resultan útiles en el contexto de su pensamiento antropológico. Esto significa que el filósofo judeohelenístico no solo conocía muchos de los desarrollos científicos y médicos vigentes en su época, sino que además fue capaz de seleccionar dentro de esos campos del saber aquellos elementos teóricos que se adaptaban mejor a sus propios parámetros de pensamiento y de rechazar o ignorar aquellos que implicaban un cuestionamiento o un cambio de perspectiva respecto de los lineamientos conceptuales de su filosofía. 


\section{EDICIONES Y TRADUCCIONES}

Alamillo, Assela (trad.) [1981] (reimpr. 1992), Sófocles. Tragedias. Madrid: Gredos.

Cholodniak, Ivan (ed.) (1889), Censorinus. De die natali. St. Petersburg: Russian Imperial Academy of Sciences.

Cohn, Leopold, Wendland, Paul y Reiter, Siegfried (eds.) [1896-1915] (reimpr. 1962), Philonis Alexandrini Opera quae supersunt, vols. I-VII. Berlin: De Gruyter.

Colson, Francis H. y Whitaker, George H. (eds.) (1929-1939), Philo, vols. I-X. London-New York: Heinemann.

Dain, Alphonse y Mazon, Paul (eds.) [1958] (reimpr. 1968), Sophocle, vol. 2. Paris: Les Belles Lettres.

Diels, Hermann y KRAnz, Walther (eds.) ['61951-1952] (reimpr. 1966), Die Fragmente der Vorsokratiker, vols. 1-2. Berlin: Weidmann.

Diggle, James (ed.) (1994), Euripidis fabulae, vol. 3. Oxford: Clarendon Press.

Drossaart Lulofs, Hendrik J. (ed.) [1965] (reimpr. 1972), Aristotelis de generatione animalium. Oxford: Clarendon Press.

Grensemann, Hermann (ed. y trad.) (1968), Hippokrates. Über Achtmonatskinder. Über das Siebnmonatskind (Unecht). Corpus Medicorum Graecorum I 2, 1. Berlin: Akademie Verlag.

JEBB, Richard (trad.) (1887), The Oedipus Tyrannus of Sophocles. Cambridge: Cambridge University Press.

Joly, Robert (colab. Byl, Simon) (ed. y trad.) (2003), Hippocrate. Du Regime. Corpus Medicorum Graecorum I 2, 4. Berlin: Akademie Verlag.

Jones, William H. S. (ed. y trad.) [1931] (reimpr. 1959), Hippocrates IV: Nature of man, Regimen in health, Humours, Aphorisms, Regimen I-III, Dreams. Loeb Classical Library. London: Heinemann / Cambridge: Harvard University Press.

LiTtRé, Emile (ed.) [1831-1869] (reimpr. Amsterdam, 1961-1962), Oeuvres Complètes d'Hippocrate, 10 vols. Paris: Baillière.

Mangeart, Jacques (trad.) (1843), Censorinus. Sur le jour natal. Paris: Panckoucke.

Marcus, Ralph (trad.) [1953] (reimpr. 1993), Philo. Questions and answers on Genesis. Cambridge-London: Harvard University Press.

Martín, José Pablo (ed.) (2009-2012), Filón de Alejandría. Obras Completas, vols. I-III, V. Madrid: Trotta.

MAu, Jürgen (ed.) (1971), «Placita Philosophorum» en MAU, Jürgen (ed.), Plutarchi Moralia, vol. 5.2.1. Leipzig: Teubner: 50-153.

Murray, G. Gilbert (ed.) [21955] (reimpr. 1960), Aeschyli Tragoediae. Oxford: Clarendon Press.

PotTer, Paul (ed. y trad.) (1995), Hippocrates VIII: Places in man, Glands, Fleshes, Prorrhetic 1-2, Physician, Use of Liquids, Ulcers, Hemorrhoids and Fistulas. Loeb Classical Library. Cambridge: Harvard University Press.

Potter, Paul (ed. y trad.) (2012), Hippocrates X: Generation, Nature of the Child, Nature of Women, Barrenness, Diseases 4. Loeb Classical Library. Cambridge: Harvard University Press.

Rodríguez Blanco, M. Eugenia (trad.) (2003), «Sobre la generación. Sobre la naturaleza del niño. Sobre las enfermedades IV» en Villa Polo, Jesús, Rodríguez Blanco, M. Eugenia, 
Cano Cuenca, Jorge y Rodríguez Alfageme, Ignacio (trads.) (2003), Tratados hipocráticos, vol. VIII. Madrid: Gredos: 232-341.

Roscher, Wilhelm H. [1913] (reimpr. New York, 1967), Die hippokratische Schrift von der Siebenzahl. Paderborn: Schöningh.

SÁnchez, Ester (trad.) (1994), Aristóteles. Reproducción de los animales. Madrid: Gredos.

Sanz Mingote, Lourdes y Ochoa Anadón, José A. (trad.) (1988), Tratados Hipocráticos, vol. IV. Madrid: Gredos.

Triviño, José María (trad.) (1975-1976), Obras completas de Filón de Alejandría, 5 vols. Buenos Aires: Acervo Cultural.

Villa Polo, Jesús, Rodríguez Blanco, M. Eugenia, Cano Cuenca, Jorge y Rodríguez Alfageme, Ignacio (trads.) (2003), Tratados hipocráticos, vol. VIII. Madrid: Gredos.

Young, D. (post Ernst DienL) (ed.) (21971), Theognis. Leipzig: Teubner.

\section{BIBLIOGRAFÍA CITADA}

BAER, Richard A. (1970), Philo's use of the categories male and female. Leiden: Brill.

BrÉHIER, Émile (1950), Les idées philosophiques et religieuses de Philon d'Alexandrie. Paris: Librairie Philosophique J. Vrin.

García GonzÁlez, José Antonio (2009), «Diferenciación sexual y teorías reproductivas en época clásica», Baetica 31: 207-228.

Hogan, Larry P. (1992), Healing in the Second Temple Period. Freiburg: Universitätsverlag / Göttingen: Vandenhoeck und Ruprecht.

Joly, Robert (1976), «La structure du feetus de huit mois», AC 45, fasc. 1: 173-180.

JouAnNA, Jacques (1973), «Tradition manuscrite et structure du traité hippocratique Sur le foetus de huit mois», REG 86, fasc. 409-410: 1-16.

Laín Entralgo, Pedro (1970), La medicina hipocrática. Madrid: Revista de Occidente.

Lonie, Iain M. (1981), The Hippocratic Treatises "On Generation», "On the Nature of the Child», «Diseases IV»: A Commentary. Berlin-New York: de Gruyter.

Lloyd, Geoffrey E. R. [1983] (reimpr. Oxford, 1986), Science, folklore and ideology. Studies in the Life Sciences in Ancient Greece. Cambridge: Cambridge University Press.

Longrigg, James (1993), Greek rational medicine. Philosophy and medicine from Alcmaeon to the Alexandrians. London-New York: Routledge.

Martín, José Pablo (2009), «Introducción general», en Martín, José Pablo (ed.), Filón de Alejandría. Obras Completas, vol. I. Madrid: Trotta.

Mattila, Sharon L. (1996), «Wisdom, Sense Perception, Nature, and Philo's Gender Gradient», HThR 89/2: 103-129. Doi: http://dx.doi.org/10.1017/S0017816000031941.

Sty, Dorothy I. (1996), Philo's Alexandria. London: Routledge. 